\title{
Secção da aorta descendente por traumatismo fechado do tórax: tratamento cirúrgico com sucesso
}

Francisco GREGORI Jr.*; Roberto TAKEDA**; Osney MOURE ${ }^{\star \star}$; Samuel S. SILVA**; José ISPER ${ }^{\star \star *}$; Walace K. AQUINO**; Thelma E. FERREIRA***, Amilcar MOCELIN ${ }^{\star \star \star}$; Eduardo SAHÃO**, Marcos P. GOULART**

RBCCV $44205-70$

GREGORI Jr., F.; TAKEDA, R.; MOURE, O.; SILVA, S. S.; ISPER, J.; AQUINO, W. K.; FERREIRA, T. E.; MOCELIN, A.; SAHẢO, E.; GOULART, M. P. - Secçâo da aorta descendente por traumatismo fechado do tórax: tratamento cirúrgico com sucesso. Rev. Bras. Cir. Cardiovasc., 3(3):216-219, 1988.

RESUMO: É descrito caso de secção da aorta torácica descendente, após a emergência da artéria subclávia esquerda decorrente de trauma fechado do tórax. O diagnóstico precoce e o rápido tratamento cirúrgico foram fundamentais para a excelente evolução pós-operatória. 0 emprego de circulação extracorpórea átrio esquerdo-femoral para a interposição de tubo de Dacron restabelecendo o fluxo arterial, foi fundamental para a prevenção de paraplegia por lesão da medula espinhal já levemente isquêmica.

DESCRITORES: traumatismos do tórax; rotura de aorta, cirurgia.

\section{INTRODUÇÃO}

As lesōes da aorta torácica descendente, secundárias a traumatismo fechado do tórax, têm aumentado de freqüência, em nosso meio, especialmente pelo elevado número de acidentes automobilísticos. Aproximadamente $85 \%$ dos pacientes morrem logo após o acidente ${ }^{1.2}$. Dos $15 \%$ restantes, a metade falece nas primeiras 48 horas, quando submetidos a tratamento clínico ${ }^{3}$. A rapidez e a agressividade no tratamento cirúrgico são fatores importantes para a sobrevida do paciente e previnem as complicaçōes que costumam advir. A lesão mais grave é a secção da aorta e a paraplegia, a complicação mais temida, secundária à isquemia da medula espinhal decorrente da hipoperfusão, devido ao trauma, choque e também ao método cirúrgico empregado. Relatos, na literatura, têm chamado a atenção para a gravidade dessas lesōes, tendo as estatísticas apresentado resultados surpreendentes $^{1-7}$. Nāo existem publicaçōes nacionais mostrando sucesso cirúrgico, em casos de secção total da aorta descendente por trauma fechado do tórax.

O objetivo deste estudo é apresentar o caso de paciente com esse tipo de lesão, operada com auxilio da circulação extracorpórea átrio esquerdo-artéria femoral, com excelente evolução pós-operatória, que computamos ao precoce diagnóstico e tratamento adotados.

\section{DESCRIÇÃO DO CASO}

J.I.M., paciente.feminina, com 33 anos de idade, vítima de acidente automobilistico, foi internada no Pronto Socorro, com contusão da parede anterior do tórax.

\footnotetext{
Trabalho realizado no Serviço de Cirurgia Cardiaca da Faculdade de Medicina da Universidade Estadual de Londrina e no Hospital Evangélico de Londrina. Londrina, PR, Brasil.

Recebido para publicaçăo em 10 de dezembro de 1988

- Da Faculdade de Medicina da Universidade Estadual de Londrina e do Hospital Evangélico de Londrina.

*. Do Hospital Evangélico de Londrina.

.. Da Faculdade de Medicina da Universidade Estadual de Londrina

Endereço para separatas: Francisco Gregori Jr. Av. Bandeirantes, 994. 86015 Londrina, PR, Brasil
} 
GREGORI Jr., F.; TAKEDA, R.; MOURE, O.; SILVA, S. S.; ISPER, J.; AQUINO, W. K.; FERREIRA, T. E.; MOCELIN. A.; SAHĀO.

E.; GOULART, M. P. - Seç̧ão da aorta descendente por traumatismo fechado do tórax: tratamento cirúrgico com sucesso. Rev. Bras. Cir. Cardiovasc., 3(3): 216-219, 1988.

A paciente estava consciente e não mostrava sinais de fraturas ósseas. Ao exame físico, apresentava hipotensão arterial, sudorese, palidez cutâneo-mucosa e os batimentos cardiacos apenas com aumento da freqüência. O estado clínico melhorou com administração de sangue.

O estudo radiológico do tórax (Figura 1) mostrava alargamento do mediastino com borramento de botão aórtico, deslocamento da veia cava superior para a direita, do brônquio fonte esquerdo em sentido caudal, ausência de hemotórax e de fratura de arcos costais. O eletrocardiograma era normal.

Foi realizada aortografia via artéria femoral direita, sendo impossível a progressão do cateter até o botão aórtico. Nesta altura, foi realizada injeção de contraste, mostrando aortograma em fundo cego (Figura 2). Realizada aortografia anterógrada via artéria braquial direita, constatou-se alargamento não uniforme da aorta torácica descendente após a origem da artéria subclávia esquerda, com enchimento da aorta descendente (Figura 3).

Com o diagnóstico de rotura da aorta, a cirurgia foi indicada e realizada de imediato, com a paciente levemente hipotensa, com pulsos pediosos debilmente palpados e com formigamento dos membros inferiores. Através de toracotomia no quarto espaço intercostal esquerdo, constatou-se pequena quantidade de sangue na cavidade pleural, porém, após o afastamento do pulmão esquerdo, observou-se grande hematoma retropleural estendendo-se desde a croça da aorta até sua porção descendente, próxima ao diafragma.

Foi estabelecido by-pass átrio esquerdo-artéria femoral e a pleura foi aberta sobre o hematoma. Após cuidadosa retirada dos coágulos, houve sangramento intenso, que, após seu controle, evidenciou-se secção transversa total da aorta (Figura 4) a $1,5 \mathrm{~cm}$ da emergência da artéria subclávia esquerda estando o coto proximal da aorta distante aproximadamente $5 \mathrm{~cm}$ do coto

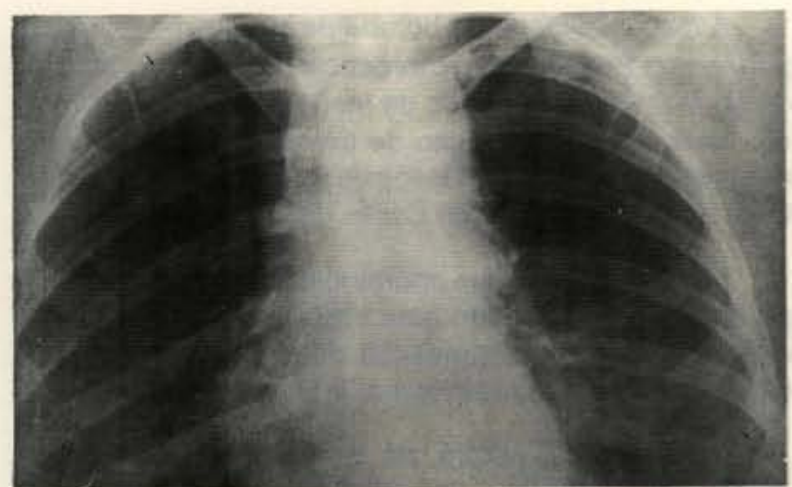

Fig. 1 - Radiografia do tórax pré-operatório. Discreto alargamento do mediastino com borramento do botão aórtico, deslocamento da veia cava superior para a direita $e$ do brônquio fonte $€$ squerdo no sentido caudal. Ausência do hemotórax e de fraturas de arcos costais

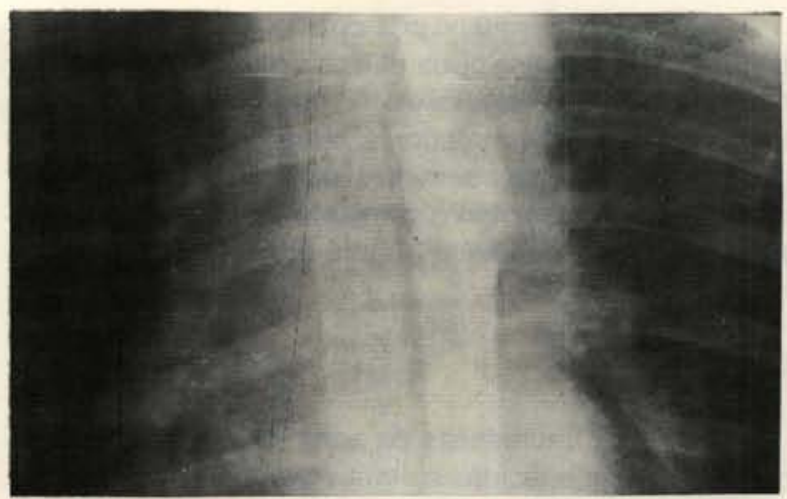

Fig. 2 - Aortografia via artéria femoral mostrando interrupção do contraste da aorta torácica descendente após enchimento de pseudo-aneurisma.

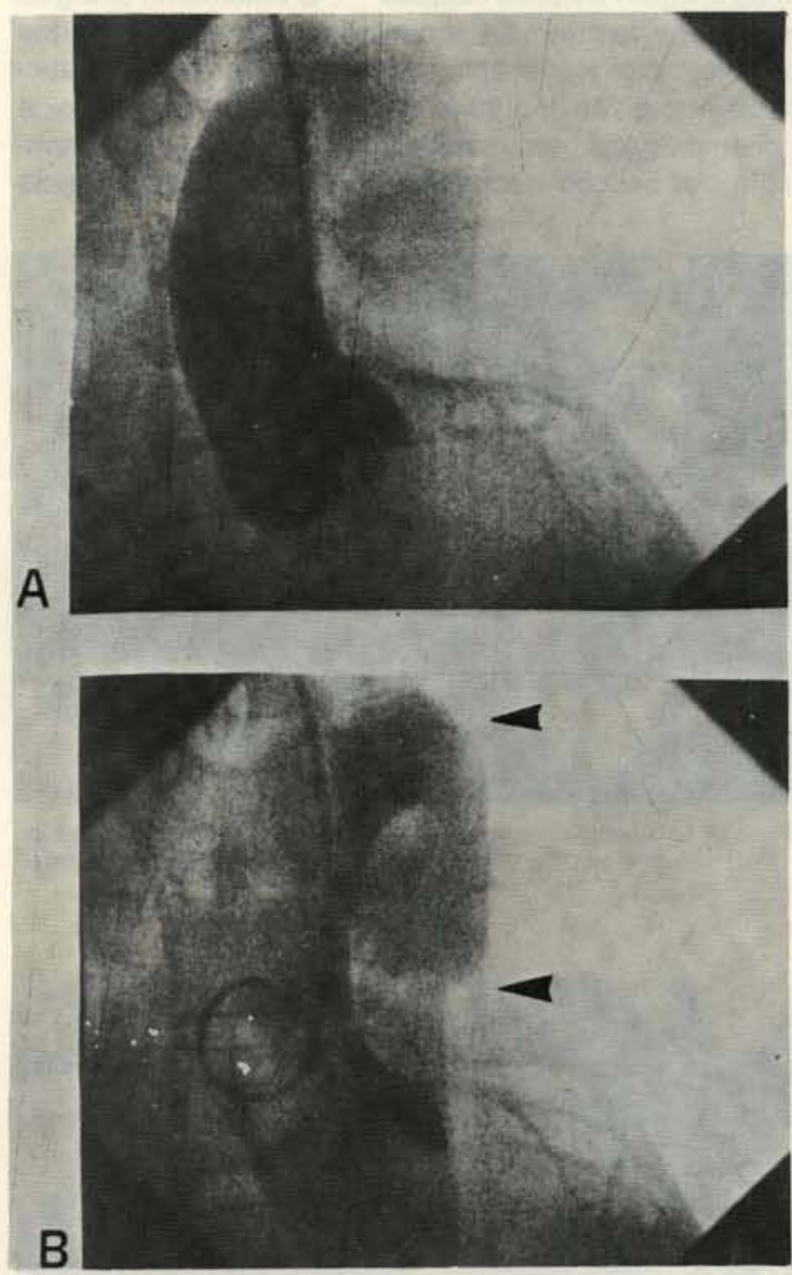

Fig. 3 - Aortograma via artéria braquial direita mostrando em $\mathrm{A}$, aorta ascendente e borramento de aorta descendente. Em B, nota-se na seta inferior, zona de secção da aorta.

distal. Um tubo de Dácron n: 16 foi interposto entre os dois cotos aórticos e o fluxo sangüíneo arterial restabelecido (Figura 5). 
GREGORI Jr., F.; TAKEDA, R.; MOURE, O.; SILVA, S. S.; ISPER, J.; AQUINO, W. K.; FERREIRA, T. E.; MOCELIN, A.; SAHĀO.

E.; GOULART, M. P. - Secção da aorta descendente por traumatismo fechado do tórax: tratamento cirúrgico com sucesso. Rev. Bras. Cir. Cardiovasc., 3(3): 216-219, 1988.

A paciente evoluiu no pós-operatório sem complicaçōes. Ao exame clínico, os pulsos eram bem palpados, tanto radiais como pediosos. A motilidade e a sensibilidade dos membros inferiores eram normais. A aortografia, realizada três semanas após a alta hospitalar, mostrou restabelecimento completo do fluxo sangüineo para a aorta descendente (Figura 6).

\section{COMENTÁRIOS}

As lesões traumáticas da aorta torácica são entidades bastante conhecidas e, freqüentemente, associadas a acidentes do trânsito. Uma em cada cinco a seis mortes por acidente automobilístico é devida à rotura da aorta descendente ${ }^{4}$. É estimado que apenas $10-20 \%$ das vítimas chegam vivas ao hospital ${ }^{4,5}$. O tratamento cirúrgico é indicado de urgência, sendo a interposição de enxertos plásticos o procedimento mais empregado para restabelecimento do fluxo arterial. No nosso caso, essa técnica foi empregada para restabelecer a luz aórtica, interrompida por secção transversa, logo após a emergência

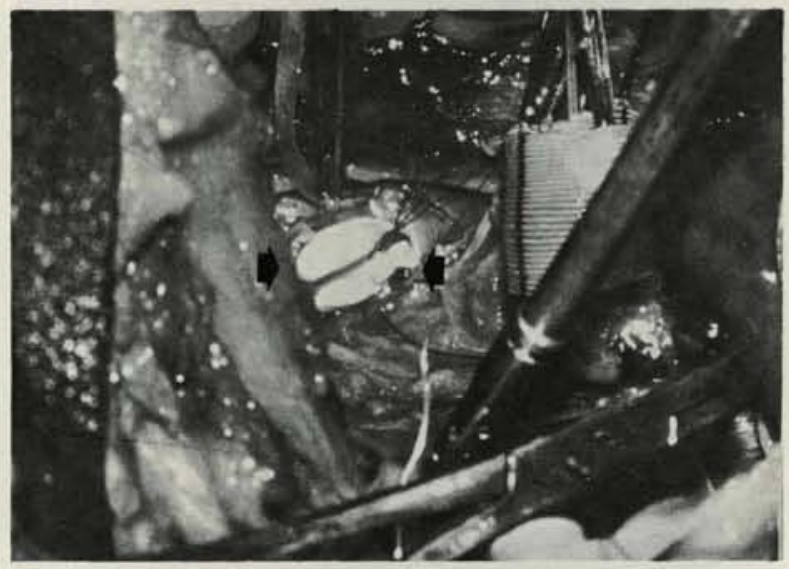

Fig. 4 - Foto operatória mostrando aorta torácica descendente seccionada e tubo de Dacron para ser implantado no coto proximal (seta)

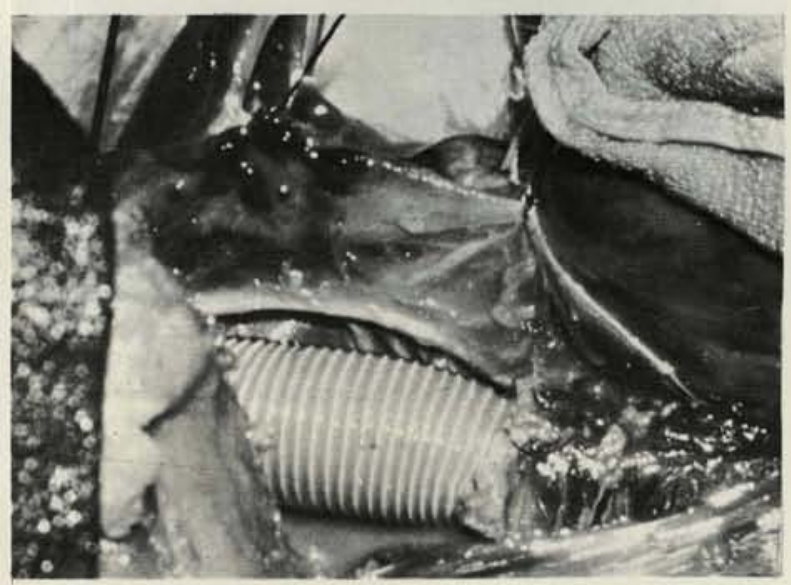

Fig. 5 - Tubo de Dacron interposto na aorta descendente.

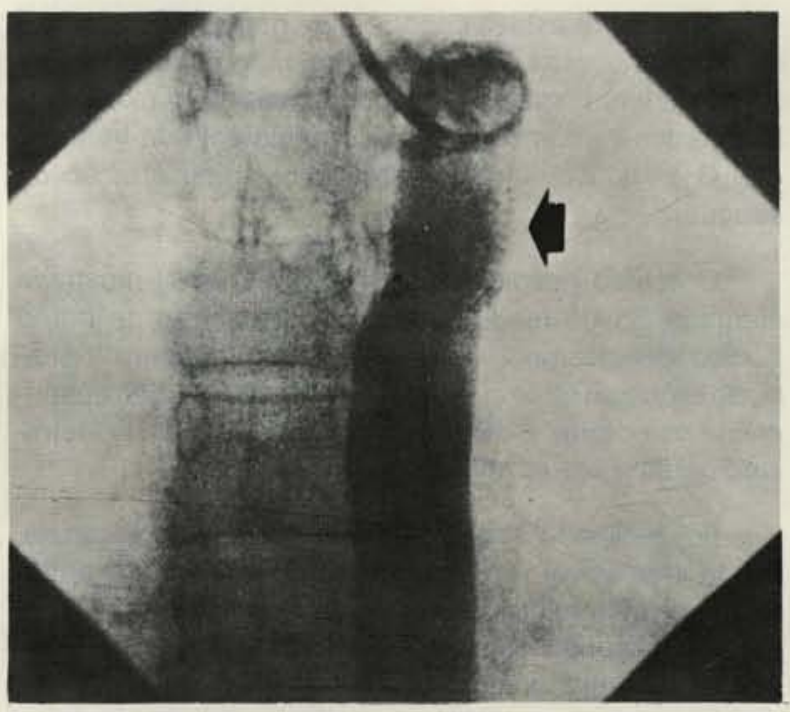

Fig. 6 - Aortograma pós-operatório mostrando restabelecimento da continuidade da aorta torácica descendente por fubo de Dacron. (seta)

da artéria subclávia esquerda. Aliás, é nesse local da aorta que a maioria das lesōes ocorre, próximas ao ligamento arterioso, que, com o trauma, fixa a aorta que se rompe quando da desaceleração brusca. $O$ afastamento de $5 \mathrm{~cm}$ de extensão entre as duas bocas aórticas, observado no nosso caso, nos chamou a atenção, só não falecendo a paciente dado que a pleura parietal nāo se rompeu e o fluxo arterial manteve-se através de um falso aneurisma. A dissecção cuidadosa da regiâo somente foi possivel pelo estabelecimento de by-pass átrio esquerdo-artéria femoral. Todos os ramos intercostais foram poupados e a isquemia transitória observada no pré-operatório reverteu-se, após a cirurgia.

Os resultados com o tratamento cirúrgico sāo, geralmente, como no nosso caso, excelentes, desde que instituido precocemente ${ }^{7}$. Não fossem as lesões associadas em outros órgãos, a mortalidade hospitalar seria mais baixa.

A disponibilidade da bomba de circulação extracorpórea, nos Serviços de emergência, é fundamental. Pode-se resolver esses tipos de lesōes com sutura direta, ou, mesmo, com utilização de tubos, sem by-pass, porém, algumas vezes, em lesões extensas, é mais confortável e seguro o emprego desse dispositivo.

Mais importante que aparelhos, no entanto, é a equipe cirúrgica estar atenta para esse tipo de lesão, especialmente quando na presença de alargamento do mediastino, ao estudo radiológico do tórax.

Nem sempre, porém, há tempo para investigação diagnóstica; inúmeros pacientes chegam agônicos ao hospital, sendo submetidos a toracotomia mesmo em sala de emergência. Obviamente, nesses casos, a mortalidade operatória ${ }^{7}$ é elevada, porém a operação é medida salvadora extrema. 
GREGORI Jr., F.; TAKEDA, R.; MOURE, O.; SILVA, S. S.; ISPER, J.; AQUINO, W. K.; FERREIRA, T. E.; MOCELIN, A.; SAHĀO,

E.; GOULART, M. P. - Secção da aorta descendente por traumatismo fechado do tórax: tratamento cirúrgico com sucesso.

Rev. Bras. Cir. Cardiovasc., 3(3): 216-219, 1988.

Assim, é chamada a atenção para a possibilidade de tratamento cirúgico efetivo para esse tipo de lesão, comum nos traumas torácicos após acidentes de trân- sito, possibilitando que grande número de vítimas possa ser beneficiado.

RBCCV $44205-70$

GREGORI Jr., F.; TAKEDA, R.; MOURE, O.; SILVA, S. S.; ISPER, J.; AQUINO, W. K.; FERREIRA, T. E.; MOCELIN, A.; SAHĀO, E.; GOULART, M. P. - Resection of descending aorta due to thoracic trauma: successful surgical treatment. Rev. Bras. Cir. Cardiovasc., 3(3): 216-219, 1988.

ABSTRACT: The case of a 33 year-old woman victim of an automobile accident whith a descending aorta transection due to thoracic blunt trauma surgically treated in described. The early diagnosis and treatment were responsible for the excellent postoperative results.

DESCRIPTORS: thoracic trauma; aorta rupture, surgery.

\section{REFERÊNCIAS BIBLIOGRÁFICAS}

1 PARMLEY, L. F.; MATTINGLY, W. T.; MANION, W. C.; JANKE Jr., R. J. - Non-penetrating traumatic injury of the aorta. Circulation, 17(6): 1086-1101, 1958.

2 MATTOX, K. L. - Invited commentary on blunt injury to the descending thoracic aorta. World J. Surg., 4:551, 1980.

3 PLUME, S. \& DE WEESE, J. A. - Traumatic rupture of the thoracic aorta. Arch. Surg., 114(3): 240-243, 1979.

4 SCHMIDT, C. A. \& JACOBSON, J. E. - Thoracic aortic injury: a ten-year experience. Arch. Surg., 119(11): 1244-1246, 1984.

5 MATTOX, K. L.; HOLZMAN, M.; PICKARD, L. R.; BEALL Jr., A. C.; DE BAKEY, M. E. - Clamp/repair: a safe technique for treatment of blunt injury to the descending thoracic aorta. Ann. Thorac. Surg., 40(5): 456-463, 1985.
6 MCBRIDE, L. R.; TIKID, S; STOTHERT, J. C.; BARNER, H. B.; KAISER, G. C.; WILLMAN, V. L.; PENNINGTON, D. G. - Primary repair of traumatic aortic disruption. Ann. Thorac. Surg., 43(1): 65-67, 1987.

7 MAVROUDIS, C.; ROON, A. J.; BAKER, C. C.; THOMAS, A. N. - Management of acute cervicothoracic vascular injuries. J. Thorac. Cardiovasc. Surg., $80(3)$ : 342-349, 1980.

8 MARQUES, E.; RIBEIRO, M. P.; KYRILLOS, E.; BRUM, J. M. G.; TSUZUTA, S.; ARMELIN, E.; MELO, C. P.; ZERBINI, E. J. - Derivaçāo ventrículo esquerdo-femoral nos traumatismos e aneurismas complicados da aorta descendente. J. Bras. Med., 31 (Ed. esp.): 26-28, 1976 .

9 BUFFOLO, E.; SUCCI, J.E.; GALUCCI, C. - Traumatismos Torácicos. São Paulo, Panamed, 1982. 